\title{
Hydrophilic Modification of Poly(ethylene oxide) by UV Irradiation
}

\author{
Gwang-hoe Koo and Jinho Jang \\ Department of Nano-Bio Textile Engineering, Kumoh National Institute of Technology Kumi 730-701, Korea
}

(Received: September 16, 2009/Revised: October 20, 2009/Accepted: October 21, 2009)

\begin{abstract}
Films of Poly(ethylene oxide), PEO, were modified to impart hydrophilicity via UV irradiation. The UV irradiation treatment produced new photo-oxidized groups of carbonyl and ether groups as indicated in ATR and ESCA analysis. It was found that water contact angle decreased from $15^{\circ}$ to $10^{\circ}$ and total surface energy of PEO increased from $54.2 \mathrm{mN} / \mathrm{m}$ to $76.6 \mathrm{mN} / \mathrm{m}$ with increasing UV energy, which was attributed to significant contribution of acid base interaction of the photo-oxidized PEO rather than nonpolar interaction originating from the dominant increase in Lewis acid parameter. The increased hydrophilicity and surface energy were also proved by the decreased water wetting time.
\end{abstract}

Keywords: Poly(ethylene oxide), UV irradiation, photo-oxidation, surface energy, hydrophilicity

\section{Introduction}

In the biomedical applications of natural and synthetic polymers, the biocompatibility of the materials is very important, which involves the interactions between polymers and body materials of living body tissues, body chemicals, and body fluids including blood. Especially, polymers contact with blood in various implants and extracorporeal devices, requiring the polymer to be biocompatible with blood to prevent blood clotting ${ }^{1}$. Therefore polymer surface property such as a hydrophilicity or hydrophobicity should be modified according to the requirements for specific biomedical applications.

Poly(ethylene oxide), PEO, is a biocompatible, nonionic, water-soluble polymer of considerable industrial significance. PEO is highly water soluble and has a good structural affinity with the water molecules, which explains the presence of strong hydrogen bonding between the ether oxygen atom and hydrogen atoms of the water molecules ${ }^{2}$.

PEO forms an important class of new biomaterials with a number of applications. Several studies related with PEO applications have been carried out including ion-beam modification of PEO based polymer electrolytes ${ }^{3)}$, self-assembled hydrogels formed by biodegradable PEO-PHB-PEO triblock copolymers and $\alpha$-cyclodextrin for controlled drug delivery system ${ }^{4)}$, gamma radiation synthesis of superabsorbent $\mathrm{PEO} /$ tragacanth hydrogel ${ }^{5}$, , the preparation of physically stabilized anti-fouling film for biomedical

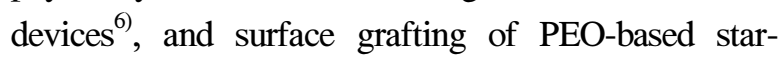
shaped molecules for bioanalytical and biomedical application $^{7)}$.

Synthetic polymers often require high degree of multi-functional properties as well as inherent properties, which are closely related with surface properties such as wettability, antistaticity, antimicrobial property, and biocompatibility, and so on. Therefore, various surface modification methods are in use to change relatively inert polymer surfaces. UV irradiation has become increasingly popular due to enhanced lamp intensity and versatile applications comparable to corona, plasma, electron beam, gamma-irradiation treatment.

In case of hydrophobic polymers, several surface modification studies by UV irradiation have been carried out including cationization of PET fabrics by the photografting of cationic monomers ${ }^{8}$, tone-ontone dyeing of PET fabrics by selective UV irradiation",

${ }^{\dagger}$ Corresponding author. Tel: +82-54-478-7715; Fax: +82-54-478-7710; e-mail: jh.jang@kumoh.ac.kr 
preferential face-coating of PET knit for water and oil repellent finish ${ }^{10)}$, and improvement of hydrophilicity and dyeability on the modified PLA films ${ }^{11)}$.

This study is to investigate the effects of UV irradiation on surface properties of PEO including hydrophilicity, surface energy, and chemical composition using various surface analysis such as ATR, ESCA, and contact angle measurement.

Also wettability was assessed by the wetting time of water on the PEO surface.

\section{Experimental}

\subsection{Materials}

Polyethylene oxide (PEO) powder with a $\mathrm{Mv}_{\mathrm{v}}$ of 300,000, supplied by Aldrich, was used for the study.

\subsection{Film preparation}

PEO powder was thoroughly spread on a dismountale mold. The mold was pressed between two heated plates at $200^{\circ} \mathrm{C}$ for a few minutes under a pressure of $10 \mathrm{MPa}$. The mold was allowed to cool under pressure to ambient temperature.

The thickness of the films was measured about 200 $\mu \mathrm{m}$. The irradiation was carried out with a continuous UV irradiator containing a D-bulb of $80 \mathrm{~W} / \mathrm{cm}$.

UV energy was adjusted to find optimal surface modification condition for the PEO films by changing UV irradiation time.

\subsection{Surface characterization}

PEO films were measured by an ATR (A537-L11) apparatus attached with a $\mathrm{ZnSe}$ crystal using a Tensor 27 spectrophotometer (Bruker Optics). The subtracted spectra were obtained by deducting the absorbance of the untreated film from that of the treated after standardization.

ESCA (Electron Spectroscopy for Chmical Analysis) was performed on the surface of the PEO films using a X-ray photoelectron spectroscope (ThermoFisher). The element ratio of $\mathrm{C}_{1 \mathrm{~s}}$ peaks to $\mathrm{O}_{1 \mathrm{~s}}$ peaks was calculated and the $\mathrm{C}_{1 \mathrm{~s}}$ peaks was deconvoluted by assigning the binding energy of C-C/C-H, R-C-O, and C=O bonds to $285.3 \mathrm{eV}, 285.9$ $\mathrm{eV}$, and $286.4 \mathrm{eV}$ respectively. The nonlinear least squares fitting (NLLSF) program with a GaussianLorentzian product function was used for curvefitting of the $\mathrm{C}_{1 \mathrm{~s}}$ spectra. The surface chemical composition was calculated from the areas of the relevant spectral peaks.

\subsection{Contact angle measurement and surface energy calculation}

Static goniometer attached with a CCD camera (Phoenix 300, Ahtech) was used to measure contact angles of water, diiodomethane and glycerin on UV irradiated samples by sessile drop method under constant temperature and humidity condition $\left(20^{\circ} \mathrm{C}\right.$, $65 \% \mathrm{RH})$.

Three measurements or more were averaged to calculate the surface energy of the PEO film according contact angles on the samples. Surface energy was calculated according to the method of van Oss et $a l^{12)}$ using the contact angles of the three liquids. When a liquid wets on a solid surface, Young's equation and work of adhesion $\left(\mathrm{W}_{\mathrm{a}}\right)$ are as follows:

$$
\begin{gathered}
\gamma_{L}(\cos \theta)=\gamma_{S}-\gamma_{S L} \\
W_{a}=\gamma_{S}+\gamma_{L}-\gamma_{S L}
\end{gathered}
$$

where $\gamma_{S}, \gamma_{L}$ and $\gamma_{S L}$ are interfacial energies of solid/air, liquid/air and solid/liquid interfaces respectively.

The total surface energy $\left(\gamma_{S}^{T O T}\right)$ of a solid surface is the sum of the Lifshitz van der Waals component $\left(\gamma_{S}^{L W}\right)$ and the Lewis acid-base component $\left(\gamma_{S}^{A B}\right)$ of which the latter is the geometric sum of the electron-withdrawing parameter $\left(\gamma^{+}\right)$and the electron-donating parameter $\left(\gamma^{-}\right)$.

$$
\gamma_{S}^{A B}=2 \sqrt{\gamma^{+} \gamma^{-}}
$$

Therefore the total surface energy of a solid can be calculated by the measured contact angles $(\theta)$ of the three liquids according to the following equation.

$$
\begin{aligned}
& W_{a}=\gamma_{L}(1+\cos \theta) \\
& =2 \sqrt{\gamma_{S}^{L W} \gamma_{L}^{L W}}+2\left\{\sqrt{\gamma_{S}^{+} \gamma_{L}^{-}}+\sqrt{\gamma_{S}^{-} \gamma_{L}^{+}}\right\}
\end{aligned}
$$




\subsection{Wetting time test}

Water wetting time on the modified films were measured to assess its wettability of the modified surfaces. The wetting time was the time that a drop $(25 \mathrm{mg})$ of water was totally absorbed into the film. Each sample was measured by three times or more to make an average.

\section{Results and discussion}

\subsection{Surface characterization of modified PEO films}

Surface chemical structure of the UV-irradiated PEO was investigated by the ATR analysis (Fig. 1 and 2). In case of untreated PEO film, the stretching bands of methylene C-H located at 2920 and $2856 \mathrm{~cm}^{-1}$, the bending of the $\mathrm{C}-\mathrm{H}$ appeared at 1381 $\mathrm{cm}^{-1}$, and the C-O vibration was found at $1083 \mathrm{~cm}^{-1}$.

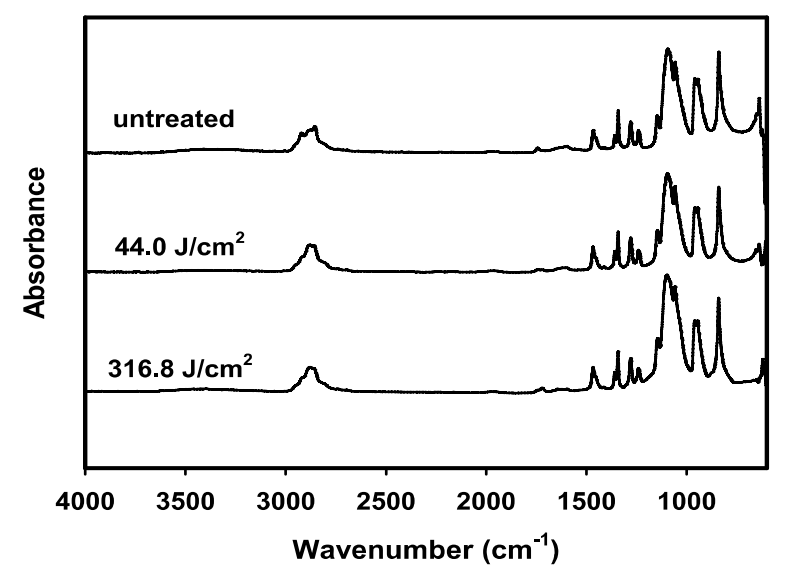

Fig. 1. ATR spectra of UV-irradiated PEO films.

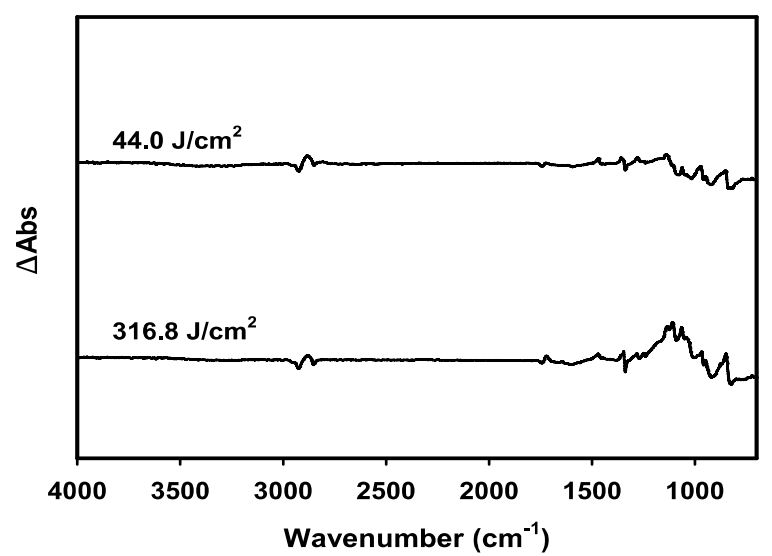

Fig. 2. Subtracted ATR spectra of UV-irradiated PEO films.
Whereas the methylene stretching of the untreated PEO disappeared upon UV irradiation, new stretching of a methine C-H peak appeared at $2883 \mathrm{~cm}^{-1}$ and the $\mathrm{C}=\mathrm{O}$ bonds were intensified at $1722 \mathrm{~cm}^{-1}$, presumably due to the photo-oxidation of the PEO surface.

When the PEO surface was analyzed by ESCA, the photo-oxidation of PEO surface was again substantiated by the change in chemical compositions of the UV-irradiated PEO films (Table 1 and Fig. 3).

Table 1. Chemical compositions of UV-irradiated PEO films

\begin{tabular}{|c|c|c|c|c|c|c|}
\hline \multirow{2}{*}{$\begin{array}{c}\text { UV } \\
\text { energy } \\
\left(\mathrm{J} / \mathrm{cm}^{2}\right)\end{array}$} & \multicolumn{4}{|c|}{$\mathrm{C}_{\mathrm{ls}}(\%)$} & \multirow{2}{*}{$\begin{array}{l}\mathrm{O}_{1 \mathrm{~s}} \\
(\%)\end{array}$} & \multirow{2}{*}{$\begin{array}{c}\mathrm{O}_{\mathrm{ss}} / \mathrm{C}_{\mathrm{ls}} \\
(\%)\end{array}$} \\
\hline & $\mathrm{C}-\mathrm{C} / \mathrm{C}-\mathrm{H}$ & C-O-R & $\mathrm{R}_{2} \mathrm{C}=\mathrm{O}$ & Total & & \\
\hline untreated & 63.1 & 2.7 & - & 69.0 & 30.9 & 44.8 \\
\hline 44.0 & 52.9 & 5.9 & 1.0 & 66.4 & 33.7 & 50.8 \\
\hline 316.8 & 53.4 & 5.3 & 3.1 & 67.1 & 32.9 & 49.1 \\
\hline
\end{tabular}

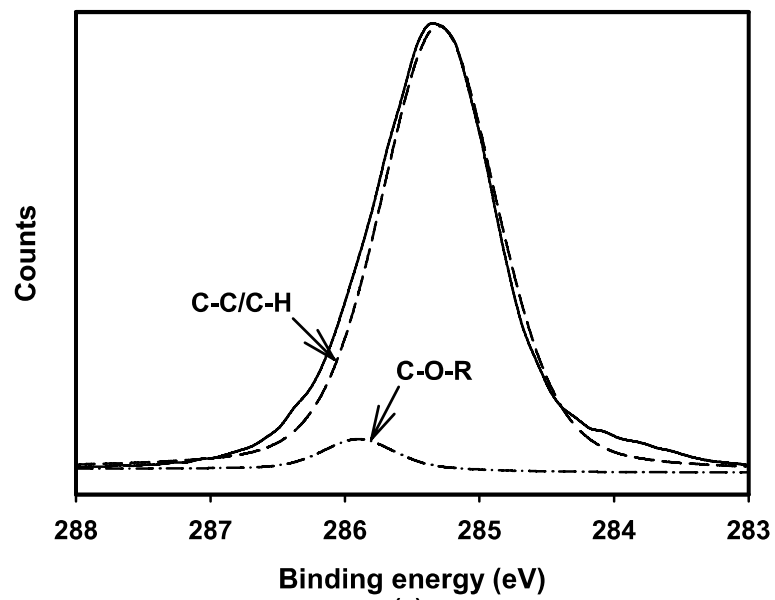

(a)

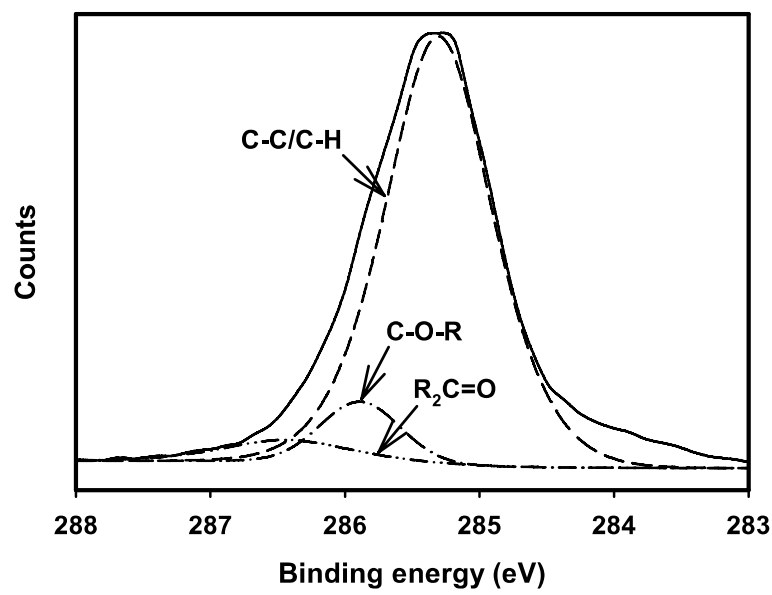

(b)

Fig. 3. Curve fitting of $C_{1 s}$ spectra of (a) untreated and (b) UV-irradiated PEO films. 
The $\mathrm{O}_{1 \mathrm{~S}}$ content increased and $\mathrm{C}_{1 \mathrm{~S}}$ decreased with UV irradiation compared to the untreated PEO surface, resulting in higher $\mathrm{O}_{1 \mathrm{~S}} / \mathrm{C}_{1 \mathrm{~S}}$ with UV irradiation from $44.8 \%$ to $50.8 \%$. With the curve deconvolution of the $\mathrm{C}_{1 \mathrm{~S}}$ peaks into those of individual carbon functionality, the carbonyl and ether groups seemed to be incorporated into the polymer surfaces with increasing UV irradiation.

The photo-scission and photo-oxidation of PEO may be beneficial in enhancing the hydrophilic property of the PEO.

\subsection{Surface energy of modified PEO films}

The surface energies of PEO films were assessed with the contact angles of three test liquids on the films (Table 2). The UV irradiated PEO films became more hydrophilic, as indicated in the decreased water contact angle from $15.8^{\circ}$ to $10.4^{\circ}$.

It may be resulted from the introduced hydrophilic polar groups on the photoxidized PEO surface. Surface energy components were calculated and individual values were shown in Table 3 .

The total surface energy $\left(\gamma^{T O T}\right)$ of the untreated PEO film was $54.2 \mathrm{mN} / \mathrm{m}$ consisting of a nonpolar component $\left(\gamma^{L W}\right)$ and a polar component $\left(\gamma^{A B}\right)$ having 46.5 and $7.7 \mathrm{mN} / \mathrm{m}$ respectively, where Lewis acid $\left(\gamma^{+}\right)$and base $\left(\gamma^{-}\right)$parameters are 0.2 and 69.0 $\mathrm{mN} / \mathrm{m}$ respectively.

Table 2. The contact angles of UV-irradiated PEO films

\begin{tabular}{cccc}
\hline \multirow{2}{*}{$\begin{array}{c}\text { UV energy } \\
\left(\mathrm{J} / \mathrm{cm}^{2}\right)\end{array}$} & Water & Diiodomethane & Glycerin \\
\cline { 2 - 4 } untreated & 15.8 & 21.4 & 49.8 \\
44.0 & 13.4 & 22.4 & 53.6 \\
316.8 & 10.4 & 25.5 & 63.9 \\
\hline
\end{tabular}

Table 3. The surface energy of UV-irradiated PEO films

\begin{tabular}{cccccc}
\hline UV energy & \multicolumn{5}{c}{ Surface energy $(\mathrm{mN} / \mathrm{m})$} \\
\cline { 2 - 6 }$\left(\mathrm{J} / \mathrm{cm}^{2}\right)$ & $\gamma^{L W}$ & $\gamma^{A B}$ & $\gamma^{+}$ & $\gamma^{-}$ & $\gamma^{T O T}$ \\
\hline untreated & 46.5 & 7.7 & 0.2 & 69.0 & 54.2 \\
44.0 & 46.1 & 13.5 & 0.6 & 75.9 & 59.6 \\
316.8 & 45.1 & 31.5 & 2.6 & 94.3 & 76.6 \\
\hline
\end{tabular}

While the nonpolar component decreased to 45.1 $\mathrm{mN} / \mathrm{m}$ with increasing UV energy, the $\gamma^{T O T}$ increased remarkably to $76.6 \mathrm{mN} / \mathrm{m}$ due to the significant increase in the $\gamma^{A B}$ to $31.5 \mathrm{mN} / \mathrm{m}$ which was mainly resulted from substantial increase in the $\gamma^{+}$rather than that in the $\gamma^{-}$. The thirteen-fold increase in Lewis acid parameter of the UV-irradiated PEO films can be attributed to electron accepting capability of the photooxidized hydrophilic groups such as $\mathrm{C}=\mathrm{O}$ and $\mathrm{C}-\mathrm{O}$ introduced by the UV irradiation.

\subsection{Wetting times of modified PEO films}

The wettability of the irradiated PEO films was also determined by water wetting time as shown in Fig. 4. Wetting time deceased remarkably from 35.8 seconds to 12.7 seconds with increasing UV energy, which can be expected from increased hydrophilicity and surface energy of the modified PEO surface. The improved hydrophilicity of the modified PEO films may be used for the enhanced interactions of the polymer surface with body materials.

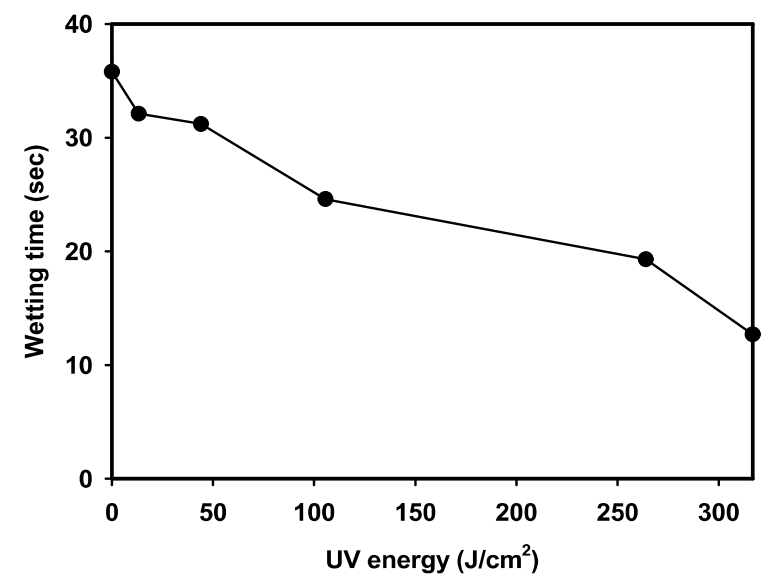

Fig. 4. Water wetting times of UV-irradiated PEO films.

\section{Conclusions}

When press-molded PEO films were modified by the UV irradiation under ambient condition, the PEO films were photo-oxidized with increasing UV energy. The UV irradiation resulted in the scission of the main chain and increase in oxygen content on the PEO surface, owing to the introduction of carbonyl and ether groups. The surface energy of the PEO 
films increased substantially due to the significantly enhanced Lewis acid parameter and hence acid-base interaction of the surfaces with increase in UV energy. Also PEO films were modified to be hydrophilic as indicated by substantially decreased contact angle and wetting times of water.

\section{Acknowledgement}

This work was supported by Research Fund, Kumoh National Institute of Technology.

\section{References}

1. S. G. Hamid, "Handbook of Polymer Degradation", Marcel Dekker, New York, USA, pp.485-513, 2000.

2. S. Dumitriu, "Polymeric Biomaterials", Marcel Dekker, New York, USA, p.253, 2000.

3. D. Singh, A. Saxena, S. Pandey, S. K. Tomar, K. Asokan, D. Kanjilal, R. Kumar and B. Bhattacharya, Ion-beam Modification of PEO Based Polymer Electrolytes, Macromol. Symp., 277, 8-13(2009).

4. J. Li, X. Li, X. Ni, X. Wang, H. Li and K. W. Leong, Self-assembled Supramolecular Hydrogels Formed by Biodegradable PEO-PHB-PEO Triblock Copolymer and $\alpha$-Cyclodextrin for Controlled Drug Delivery, Biomaterials, 27, 4132-4140(2006).

5. F. Khoylou and F. Naimian, Radiation Synthesis of Superabsorbent Polyethylene Oxide/Tragacanth Hydrogel, Radiation Physics and Chemistry, 78, 195-198(2008).
6. M. Manso-Silvan, A. Valsesia, D. Gilliland, G Ceccone and F. Rossi, Ion-beam Treatment of PEO; Towards a Physically Stabilized Antifouling Film, Surf. Interface Anal., 36, 733736(2004)

7. P. Gasteier, A. Reska, P. Schulte, J. Salber, A. Offenhausser, M. Moeller and J. Groll, Surface Grafting of PEO-Based Star-Shaped Molecules for Bioanalytical and Biomedical Applications, Macromol. Biosci., 7, 1010-1023(2007).

8. J. A. Son and J. Jang, Cationization of PET Fabrics via Continuous Photografting of [3(Methacryloylamino)propyl] trimethylammonium Chloride, Text. Sci. Eng., 44, 312-318(2007).

9. J. A. Son and J. Jang, Tone-on-tone Dyeing of PET Fabrics Using Selective UV Irradiation and Cationic Dyes, Text. Sci. Eng., 44, 142-148 (2007).

10. H. S. Lee, W. S. Lyoo and J. Jang, Effect of UV Irradiation on Cellulose Degradation of Cellulose Acetate Containing $\mathrm{TiO}_{2}$, Fiber. Polym., 8, 19 24(2007).

11. G. H. Koo and J. Jang, Surface Modification of Poly(Lactic Acid) by UV/Ozone Irradiation, Fiber. Polym., 9, 674-678(2008).

12. C. J. van Oss, R. J. Good, and M. K. Chaudhury, Additive and Nonadditive Surface-tension Components and the Interpretation of Contact Angles, Langmuir, 4, 884-891(1988). 